Copyright (C1996, American Institute of Aeronautics and Astronautics, Inc.

AIAA Meeting Papers on Disc, July 1996

A9637202, AIAA Paper 96-3068

\title{
Antiproton catalyzed fusion propulsion for interplanetary missions
}

\author{
B. N. Cassenti \\ United Technologies Research Center, East Hartford, CT \\ T. Kammash \\ Michigan Univ., Ann Arbor \\ D. L. Galbraith \\ Michigan Univ., Ann Arbor
}

\begin{abstract}
AIAA, ASME, SAE, and ASEE, Joint Propulsion Conference and Exhibit, 32nd, Lake Buena Vista, FL, July 1-3, 1996
\end{abstract}

\begin{abstract}
Interplanetary trips using chemical propellants require years to complete. A recently completed study on an antiproton catalyzed fusion reaction propulsion system has shown that the specific impulses that can be obtained are between 1500 seconds for a contained system to over 100,000 seconds for a system that directly uses the fusion reaction products. Thrust-to-weight ratios exceeding one can be sustained. This allows considerably shorter solar system travel times than conventional chemical propellants. Missions considered range from inner to outer solar system distances. A trade-off can be made between reducing travel time and reducing initial mass in low Earth orbit. Missions to the inner planets can be shortened considerably for a given mass ratio, while missions to the outermost planets will be several weeks in duration. (Author)
\end{abstract}




\section{ANTIPROTON CATALYZED FUSION PROPULSION FOR INTERPLANETARY MISSIONS}

\author{
B. N. Cassenti* \\ United Technologies Research Center \\ East Hartford, Connecticut 06084
}

\author{
T. Kammash and D. L. Galbraith \\ Department of Nuclear Engineering \& \\ Radiological Sciences \\ University of Michigan \\ Ann Arbor, MI 48109
}

\begin{abstract}
Interplanetary trips using chemical propellants require years to complete. A recently completed study on an antiproton catalyzed fusion reaction propulsion system has shown that the specific impulses that can be obtained are between 1500 seconds, for a contained system, to over 100,000 seconds, for a system that directly uses the fusion reaction products. Thrust-to-weight ratios exceeding one can be sustained. This allows considerably shorter solar system travel times than conventional chemical propellants. Missions considered range from inner to outer solar system distances. A trade-off can be made between reducing travel time and reducing initial mass in low earth orbit. Missions to the inner planets can be shortened considerably for a given mass ratio, while missions to the outermost planets will be several weeks in duration.
\end{abstract}

\section{Introduction}

During the last decade antiproton annihilation propulsion has been the subject of considerable research. ${ }^{1-5}$ The use of antiprotons to directly heat a propellent requires at least milligrams of antimatter to perform useful missions. ${ }^{6}$ Milligram quantities of antiprotons are well beyond current capabilities and will require substantial technological improvements before becoming a reality. ${ }^{7,8}$ Concepts for using antiprotons to catalyze fusion reactions that would require far fewer antiprotons bave recently been proposed.9-13 The approaches are based on: 1) antiproton fissioning of heavy nuclei, ${ }^{10-12}$ 2) direct antiproton heating for igniting a fusion reaction, 9,13 and 3) muon production using antiprotons for muon catalyzed fusion. ${ }^{14}$

The Lewis et al. proposal to use antiprotons to fission heavy nuclei has been the subject of considerable re- search. ${ }^{10-12}$ When an antiproton annihilates in a heavy nucleus of uranium or plutonium, the nucleus fissions almost $100 \%$ of the time. ${ }^{15}, 16$ About $20 \%$ of the fission energy is present in the fission fragments, and over one dozen neutrons are emitted. Some of these neutrons initially released will produce additional fissions with a considerably lower yield of neutrons. The energy present in the fission fragments is readily absorbed by the fusion fuel. It is more difficult to absorb the neutron kinetic energy, whereas the absorption of the gamma ray energy is very difficult. Nevertheless, antiproton annihilation provides a means for sustaining fission reactions without a critical mass of fissionable material. The fission energy then provides a means for initiating fusion reactions.

Shamatov ${ }^{1,3}$ Cassenti, 5,17 and Kammash and Galbraith ${ }^{9}$ have proposed using the annihilation of antiprotons (or antihydrogen) to initiate fusion reactions directly. When an antiproton annihilates in matter it produces mostly pions and about $5 \%$ kaons. About $60 \%$ of the pions are charged. The pions are moving at relativistic velocities (about $95 \%$ of the speed of light), and the absorption of the kinetic energy requires on the order of meters of path length for significant energy absorption. Of course, the energy must be absorbed before the pions decay (i.e., in about 20 ns) into a muon and an associated neutrino. The muons are charged and will travel on the order of kilometers before decaying. The muon has a mean life about 100 times the life of a pion. Since muons are charged they can also deposit energy in the plasma. The muon decays into an electron, or a positron, and two associated neutrinos. The electrons, or positrons, can also deposit energy in a plasma. To increase the path length in the plasma a magnetic field could be applied but the relativistic speeds require extremely large fields. For example, about $100 \mathrm{kG}$ are required for 1 -m-diam

Copyright $@ 1995$ by the American Institute of Aeronautics and Astronautics, Inc. All rights reserved.

* Associate Fellow, AIAA Senior Principal Engineer 
containment and $10 \mathrm{MG}$ for a 1-cm-diam pellet. Magnetic fields are difficult to generate in a steady state but can be generated in transient situations. Hasegaw $\mathrm{a}^{18}$ and Kammash and Galbraith ${ }^{19,20}$ have proposed using magnetic fields developed at the surface of laser heated materials. At the heated surface the material is ionized, with the ion cores and the electrons sharing the absorbed energy. The ions and electrons stream away from the surface with the electrons moving faster than the ion cores. The ions and electrons moving into the surface are readily stopped. Hence there is a net negative current flow away from the surface. The net current flow creates a magnetic field which readily contains the plasma. Measurements and models ${ }^{21-23}$ indicate that the fields are large enough to contain the annihilation products resulting when an antiproton annihilates on a nucleus, and the field is large enough to isolate the plasma from a surrounding heavy metal shell. The shell will contain the plasma due to its strength and inertia, in a manner similar to inertial confinement fusion. The antiprotons provide a lightweight mechanism for heating the plasma to fusion ignition temperatures, and, hence, are well suited for use in propulsion systems.

The last method proposed for using antiprotons as a catalyst to initiate fusion reactions uses the muons, resulting from antiproton-nucleon annihilations, to sustain muon catalyzed fusion of a deutrium-tritium mixture. ${ }^{14}$ The antiprotons provide a compact source for the muons when compared to the accelerators that have been proposed ${ }^{24}$ and, hence, are ideal for use in propulsion systems.

Each of the cited methods for using antiprotons to catalyze fusion reactions present disadvantages for use in propulsion. Pellet designs for antiproton catalyzed fission reactions require an initial compression using ion beams. ${ }^{11,12}$ The ion accelerators are heavy, and there would be a distinct advantage in eliminating them. Direct heating of the plasma to initiate a fusion reaction uses very little of the annihilation energy - about $2 \%$ (Ref. 9). Muon catalyzed fusion is effective in a narrow temperature range, at about $1200 \mathrm{~K}$ to support a resonance between the tritium and deuterium atoms in a molecule. These low temperatures will be difficult to sustain.

This paper will use a combination of antiproton induced fission and magnetically insulated inertial confinement fusion. ${ }^{25}$ Muon catalyzed fission could be utilized by injecting muons, from an antiproton annihilation, at appropriately heated points in the fusion fuel, at the correct time.

Fusion pellets are ideal for use in nuclear pulse propulsion systems. Over the last four decades nuclear pulse propulsion has been proposed in several forms. ${ }^{26-29}$
Parlos and Metzger, ${ }^{29}$ have recently shown that a 100 ton nuclear device can be contained by a $9.4 \mathrm{~m}$ radius vessel, $0.2 \mathrm{~m}$ thick. The inside of the vessel wall would be lined with molybdenum. Each detonation would ablate about $0.3 \mathrm{~cm}$ of molybdenum. The final thickness of the molybdenum would have to be about $1 \mathrm{~cm}$ for optimum performance. The vessel wall itself would consist of stainless steel reinforced with NICALON fibers. The vessel would have a mass of about 1800 metric tons. When the vessel was charged with hydrogen at one atmosphere, the specific impulse was about $1000 \mathrm{sec}$ and the thrust-to-mass ratio was about 2. The predictions of Parlos and Metzger were obtained using results from previous numerical simulations. Although these simulations are an aid in scaling, they do not allow a complete examination of the relative merits of various approaches.

A simple model has been developed ${ }^{30}$ that allows investigators to estimate various effects, and predict the performance of a contained system. Estimates from this simple model predicted a higher performance than the simulations used by Parlos and Metzger, but are still somewhat below the ideal performance (i.e., the performance without losses). The simple model was built from several models. These included: a model of the expansion of the gas from the blast, a model of the structural response of the chamber, a model of the heating of the containment chamber wall, and a performance model for the performance of the rocket.

\section{Propulsion Model Summary}

The pellet to be ignited consists of several materials The fusion fuel can be deuterium-tritium, or lithium deuteride. ${ }^{25}$ The fuel has an outer radius $R_{f}$ and an inner radius $R_{c}$ (see Fig. 1). The fuel contains a hemisphere of fissionable material such as $\mathrm{U}^{235}, \mathrm{U}^{238}$, and $\mathrm{Pu}^{239}$. The fusion fuel is surrounded by a shell consisting of a heavy metal. The outer layer of the shell should be a dense highmelting temperature material such as tungsten. The inside shell layer can be a neutron generating or reflecting material, such as uranium. The shell and fuel have a hole of diameter $d$ which is perpendicular to the flat surface of the hemisphere of fissionable material. The flat surface of the hemisphere is assumed to be a small distance $\delta$ below the surface.

A pulse of antiprotons and positrons ${ }^{13}$ is injected through the hole in the shell. The energy of the antiprotons is chosen so that the annihilation occurs at the surface of the hemisphere. The annihilation of the antiprotons ionizes the fuel above the hemisphere. Fuel ions fill the empty core of the pellet with a plasma. The electrons created by the annihilation create a current flow developing a transient magnetic field. The field is contained within the shell and traps charged particles (fission fragments, ion cores, electrons, muons, and pions). The 
charged particles heat the core of the pellet to fusion temperatures. The uranium on the inside layer of the shell generates and/or reflects some neutrons back into the fuel, while the tungsten shell contains the fusion fuel hopefully long enough for the fusion reaction to go to completion.

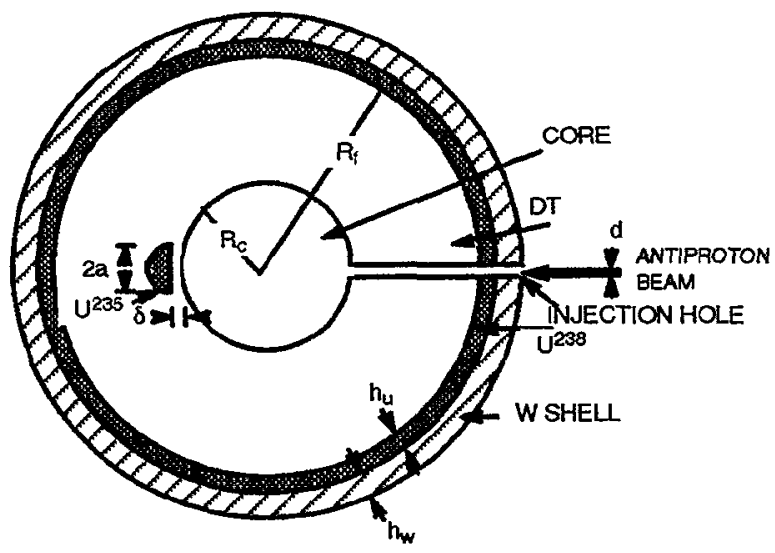

Fig. 1. Pellet construction and geometry (from Ref. 25)

The pulse of antimatter should consist of equal quantities of antiprotons and positrons to preserve neutrality..$^{13}$ The pulse can be antihydrogen or it can be partially or completely ionized.

The annihilation of the antiprotons will ionize material in the immediate neighborhood and release relativistic particles. The nonuniform, heating will create magnetic fields that can be roughly estimated as ${ }^{22}$

$$
\frac{1}{1 M G} \dot{B}=\frac{1}{10 \mathrm{ps}}\left(\frac{T}{1 \mathrm{keV}}\right)\left(\frac{10 \mu m}{d}\right)^{2} \sin \alpha
$$

and

$\frac{1}{1 M G} B=3.6\left(\frac{T}{1 \mathrm{keV}}\right)^{1 / 2}\left(\frac{10 \mu m}{d}\right)\left[\frac{A^{1 / 2}(Z+1)^{-1 / 2}}{1.1}\right] \sin \alpha$

where $\dot{B}$ is the rate of change of the magnetic field in Mega gauss, with respect to time, $\bar{B}$ is the steady magnetic field, $A$ is the atomic weight of the plasma (taken to be 2.5 for deuterium-tritium fuel), and $Z$ is the atomic number of the plasma. To transition betwsen the growth, Eq. (1) and the steady field, Eq. (2), we can take the transient field, $B(t)$ to be

$$
B(t)=\bar{B}\left(1-e^{-t h}\right)
$$

where

$v=\frac{\dot{B}}{\bar{B}}=\frac{1}{36 p s}\left(\frac{T}{1 \mathrm{keV}}\right)^{1 / 2}\left(\frac{10 \mu m}{d}\right)\left[\frac{1.1}{A^{1 / 2}(\mathrm{Z}+1)^{-1 / 2}}\right](4)$

at $\mathrm{t}=0$.

The above equations are used to describe the transient magnetic field.

The pions developed in an annihilation will deposit a small portion of their energy in the plasma, before they leave the pellet. The energy deposition, $d E / d x$, will be on the order of

$$
\frac{d E}{d x} \approx 0.5 \frac{\mathrm{MeV}}{\mathrm{cm}}\left(\frac{\varrho_{\mathrm{plassm}}}{\varrho_{\mathrm{LH}_{2}}}\right)
$$

where $\varrho_{\text {plamal }} / \varrho_{\mathrm{LH}_{2}}$ is the ratio of the plasma density to the density of liquid hydrogen. There will also be a recoil energy for annibilations in the plasma, $\varepsilon$, of about $20 \mathrm{MeV}$ (Ref. 9).

The path length over which the energy is deposited is given by

$$
s \approx 2 R_{c}
$$

if the path is helical due to the presence of a magnetic field.

The energy, $E_{\pi}$ deposited by the pions and the recoil, $\varepsilon$, is

$$
E_{\pi}=\frac{1}{2} v_{x} \frac{d E}{d x} s+\varepsilon
$$

where $v_{\pi}$ is the number of charged pions created at the annihilation site (about 3.6).

One-half of the pions move into pellet core with the plasma, and the other half move into the fission hemisphere.

When the antiproton annihilates on the flat of the hemisphere, the material will fission releasing the kinetic energy of fission fragments, $E_{f}$ and a number of neutrons and pions, which can cause additional fissions. From Ref. 12 for $U^{238}$,

$$
v_{f} \approx 13.7
$$

and

$$
E_{f} \approx 170 \mathrm{MeV}
$$

Half of the released neutrons will enter the fission hemisphere and can cause additional fissions. These additional fissions have been shown to have little effect on the performance 25 and will be neglected.

The magnetic fields decay sufficiently slowly to contain the plasma pressure until the fusion reaction be- 
gins. Hence, the confinement time, $\mathrm{t}$, can be taken as the time for a $\mathrm{p}$-wave to propagate through the thickness of the Tungsten shell. ${ }^{18}$

The $p$-wave speed in a solid is given by

$$
C_{p}=\sqrt{\frac{(1-v) E}{(1+v)(1-2 v) \varrho_{w}}}
$$

where $E$ is Young's modulus (about $4.1 \times 10^{12} \mathrm{dyne} / \mathrm{cm}^{2}$ for room temperature tungsten), $v$ is the Poisson ratio (about 0.23 for room temperature tungsten) and $\mathrm{Q}$ is the density. The inertial confinement time is bounded by

$$
t_{i}>\frac{h_{w}}{C_{p}}
$$

A rough calculation indicates that heating of the tungsten shell (e.g., due to fission gamma-ray emission) will not raise the shell temperature by more than $100^{\circ} \mathrm{C}$. Hence room temperature properties can be used. Recall that the magnetic fields will insulate the shell from the plasma.

The inertial confinement time $t_{f}$ must be greater than the time for the plasma to fuse ${ }^{18}$ where

$$
t_{f}=\frac{\sigma k}{E_{a} n_{\text {plasma }}<\sigma \nu>}
$$

where $T$ is the plasma temperature, $E_{\alpha}$ the energy of the alpha particle emitted, $n_{\text {plasma }}$ the plasma number density, $<\sigma v>$ the reaction rate, and $\mathrm{k}$ is Boltzmann's constant.

An upper unit on the plasma temperature and the core energy are related by

$$
\frac{3}{2} N k T=\frac{1}{2} N E_{a}
$$

where $\mathrm{N}$ is the number of alpha particles.

The ideal specific impulse of the system can now be determined by assuming all of the fusion alpha particle energy, from all of the fuel, is emitted with the total mass of the pellet. The exhaust velocity $v_{e}$ can then be determined from

$$
\frac{1}{2} m_{T} v_{c}^{2}=\frac{m_{f} E_{\alpha}}{2 A m_{\operatorname{amb}}}
$$

where $m_{T}$ is the total pellet mass, $m_{f}$ the total fuel mass, $m_{\mathrm{amu}}$ is the atomic mass unit and $\mathrm{A}$ is the average atomic mass of the fuel.

The specific impulse, $I_{\text {sp, }}$ can then be approximated as

$$
I_{s p}=\frac{\nu_{e}}{g}
$$

where $g$ is the acceleration of gravity at the surface of the Earth.

The model can now be used to examine specific pellet designs.

The above model was used to predict the response of a typical pellet. ${ }^{25} \mathrm{~A}$ deuterium-tritium fuel was taken for simplicity. The alpha particle energy produced in a fusion reaction was taken to be $3.5 \mathrm{MeV}$ (Ref. 18). This was the energy assumed to be present in the exhaust, since the alpha particle can be directed by magnetic fields. The reaction rate $\langle$ ov $\rangle$ was taken to be $10^{-15}$ $\mathrm{cm}^{3} / \mathrm{s}$ which occurs at a plasma temperature of $80 \mathrm{keV}$.

The pellet geometry is summarized in Table 1. The pellet has a shell of tungsten $0.01 \mathrm{~cm}$ thick, and there is no uranium on the inside of the shell. The shell has an outer radius of about $1 \mathrm{~cm}$. The hemisphere of uranium is so small that it only supports secondary fissions about $1 \%$ of the time. The antimatter pulse contains $3 \times 10^{9}$ antiprotons and lasts $30 \mathrm{~ns}$. The core density is about $1.25 \mathrm{x}$ $10^{21} / \mathrm{cm}^{3}$, if only of the material above the flat uranium surface enters the core. The core temperature is $83 \mathrm{keV}$. The magnetic field reaches about $2 \mathrm{MG}$ at end of the pulse. The tungsten shell does not move during the heating by the antimatter pulse. The characteristic inertial confinement time is at least $20 \mathrm{~ns}$, and the time for the fusion reaction is about $10 \mathrm{~ns}$. The total mass of the pellet is about $3.5 \mathrm{mg}$ and absorbs about $7.3 \times 10^{17} \mathrm{erg}$ producing a specific impulse of about $600,000 \mathrm{~s}$ for complete $(100 \%)$ fusion burning. If $10 \%$ of the fuel fuses, the ideal specific impulse is about $200,000 \mathrm{~s}$, whereas $5 \%$ yields about $150,000 \mathrm{~s}$ for an ideal specific impulse. If the pellet is used in a contained system to heat hydrogen propellant, then the specific impulse will be between 2700 and $3700 \mathrm{sec}^{30}$ depending on the propellent and ablated mass with a thrust-to-mass ratio about thirty.

Table 1 Typical pellet geometry

\begin{tabular}{ll}
\hline \multicolumn{1}{c}{ Description } & Dimension, $\mathrm{cm}$ \\
\hline Core radius, $R_{f}$ & 0.01 \\
Fuel radius, $R_{\mathrm{c}}$ & 1.0 \\
Uranium shell thickness, $h u$ & 0.0 \\
Tungsten shell thickness, $h_{u}$ & 0.01 \\
Antiproton beam radius, $d$ & 0.0003 \\
Uranium hemisphere radius, $a$ & 0.03 \\
Hemisphere distance from & 0.0075 \\
core surface, $\delta$ & \\
\hline \hline
\end{tabular}

The pellet geometry is summarized in Table 1 . Of course, if the pellet is scaled to the size of a thermonuclear warhead, then, with minor variations in design, a 
single antiproton (or neutron) will trigger the critical mass fission device resulting in nearly complete fusion. Hence, if the pellet is scaled up in size (and designed as in a thermonuclear warhead) then the pellet will ignite and fuse. The design problem is how to shrink the pellet to sizes on the order of $1 \mathrm{~cm}$. If the pellets do not contain a critical mass of uranium or plutonium, then the propulsion system will not violate the space nuclear weapon ban.

Other variations in the design are also possible. The hole in the shell and the fuel can be removed (i.e., filled) with the antiproton energy tuned to pass through the shell and fuel and then annihilate at the fissionable hemisphere. ${ }^{31}$ The tritium can be replaced with $\mathrm{Li}^{6}$ (the lithium will make the tritium upon absorbing a neutron), which will eliminate the radioactive hazard of tritium, ${ }^{1}$ and the need for a cryogenic system. Finally, plastic can be used to absorb gamma rays as in a thermonuclear warhead $^{32}$

\section{Interplanetary Trajectory Models}

Estimates for the performance of various propulsion systems can be readily obtained using the equations of celestial mechanics. There are several excellent texts that can provide the necessary background for completing performance calculations (e.g., Ref. 33).

In our celestial mechanics based models we have assumed that all maneuvers are impulse maneuvers. Hence, the time to change the velocity is much shorter than the total transit time for the mission. This is true for lower specific impulse fusion rockets (about $3000 \mathrm{sec}$ ) but may not hold when the specific impulse is near the limit for fusion propulsion (about $1,000,000 \mathrm{sec}$ ). All the planets will be assumed to be orbiting the sun in circular orbits, with all the orbits in the same plane. Finally, no use will be made of aeroassists or gravity assists.

The trajectory consists of: 1) the departure from earth orbit, 2) the transit to the planet, and 3) the insertion into a low orbit about the planet. The return trip is assumed to have the same energy requirements as the earth departure trip. Hence, only the trajectory to the planet needs to be examined. This means that the stay time at the planet could be a significant fraction of a year. The earth departure orbit and the parking orbit about the planet are both assumed to be at an altitude equal to the radius of the planet. Two types of missions will be considered: 1) minimum energy transfer (Hohmann transfer), and 2) a minimum time transfer for a given target mass ratio.

\section{Rocket Performance}

Using the assumptions above the performance of the fusion rocket can be represented by the specific impulse, Isp. The rocket equation then leads to the following relation between the mass ratio, $M R$, and the change in speed, $\Delta v$,

$$
M R=\exp \left(\frac{\Delta v}{I_{S P g_{0}}}\right)
$$

where $\mathrm{g}_{0}$ is the acceleration of gravity at the earth's surface, and the mass ratio, $M R$, is defined as the ratio of the initial mass to the final mass.

\section{Hohmann Trajectories}

Hohmann minimum energy trajectories are well known and can be readily calculated. The length of the semimajor axis, $\mathrm{a}_{0}$, of the transfer orbit is given by

$$
a_{o}=\frac{\left(r_{i}+r_{f}\right)}{2}
$$

where $r_{i}$ and $r_{f}$ are the radius the orbit of the earth and the planet about the sun, respectively. The eccentricity, $e_{0}$, of the transfer orbit is then

$$
e_{0}=\frac{\left(r_{i}-r_{f}\right)}{\left(r_{i}+r_{f}\right)}
$$

The specific angular momentum of the orbit, $h_{0}$, is

$$
h_{0}=\sqrt{P_{0} G M_{0}}
$$

where $M_{0}$ is the mass of the sun, $G$ is the gravitational constant, and

$$
P_{0}=a_{0}\left(1-e_{0}^{2}\right)
$$

The speed changes at the earth and planet require the speed of the spacecraft about the sun at the earth, $v_{i}$, and the planet $v_{f}$. These can be found from the angular momentum as

$$
\begin{aligned}
& v_{i}=\frac{h_{o}}{r_{i}} \\
& v_{f}=\frac{h_{o}}{r_{f}}
\end{aligned}
$$

The spacecraft though will have an excess hyperbolic velocity upon leaving the earth, $v_{h i}$, and upon arrival at the planet, $\mathrm{v}_{\mathrm{hf}}$, of

$$
\begin{aligned}
& v_{h i}=v_{i}-V_{i} \\
& v_{h f}=v_{f}-V_{f}
\end{aligned}
$$


where $V_{i}$ and $V_{f}$ are the circular velocities of the earth and planet about the sun, and are given by

$$
\begin{aligned}
& V_{i}=\sqrt{\frac{G M_{0}}{r_{i}}} \\
& V_{f}=\sqrt{\frac{G M_{0}}{r_{f}}}
\end{aligned}
$$

The specific energy of the hyperbolic departure and arrival orbits, $E_{i}$ and $E_{f}$ respectively, are simply

$$
\begin{aligned}
& E_{i}=\frac{1}{2} v_{h i}^{2} \\
& E_{f}=\frac{1}{2} v_{h f}^{2}
\end{aligned}
$$

The hyperbolic departure and arrival orbits have velocities at the original low earth, $v_{\mathrm{pi}}$, orbit and parking planet orbit, $\mathrm{v}_{\mathrm{pf}}$, of

$$
\begin{aligned}
& v_{p i}=\sqrt{\frac{2\left(E_{i}+G M_{i}\right)}{R_{i}}} \\
& v_{p f}=\sqrt{\frac{2\left(E_{f}+G M_{f}\right)}{R_{f}}}
\end{aligned}
$$

respectively, where $R_{i}$ is the initial earth orbit radius, and $R_{f}$ is the parking planet orbit radius, while $M_{i}$ and $M_{f}$ are the masses of the earth and the planet. The total change in speed required for the mission is now given by

$$
\Delta v=\Delta v_{i}+\Delta v_{f}
$$

where

$$
\begin{aligned}
& \Delta v_{i}=v_{p i}-\sqrt{\frac{G M_{i}}{R_{i}}} \\
& \Delta v_{f}=v_{p f}-\sqrt{\frac{G M_{f}}{R_{f}}}
\end{aligned}
$$

and $\mathrm{M}_{\mathrm{i}}$ and $\mathrm{M}_{\mathrm{f}}$ are the masses of the earth and planet respectively. The last terms in eqs. $(32,33)$ are the circular orbit speeds about the earth or planet.

Finally, the total transit time, $\Delta \mathrm{t}$, is just half the orbital period of the Hohmann transfer ellipse., or

$$
\Delta t=\pi \sqrt{\frac{a_{0}^{3}}{G M_{0}}}
$$

Equation (31) can be substituted into eq.(16) to find the mass ratio for various values of the specific impulse.
The solar system data used for the evaluation is summarized in Appendix A, and was taken from Ref. 34. The transit times are given an Table 2 , and, of course, do not depend on the specific impulse. The mass ratio required for various values of the specific impulse are given in Table 3. It can be seen that the mass ratios become approximately unity for values of the specific impulse of 30,000 seconds or more.

Table 2 Hohmann minimum energy transfer time

\begin{tabular}{lc}
\hline Planet & Time (yr) \\
\hline Mercury & 0.289 \\
Venus & 0.400 \\
Mars & 0.713 \\
Vesta & 1.085 \\
Ceres & 1.293 \\
Jupiter & 2.731 \\
Saturn & 6.048 \\
Uranus & 16.04 \\
Neptune & 30.59 \\
Pluto & 45.61 \\
\hline \hline
\end{tabular}

Minimum Transfer Time Trajectories

The trip times, though are quite large for trips to the outer solar system. Consuming more fuel could greatly reduce the trip time. Here the trip time will be minimized for a chosen mass ratio. Throughout this paper this mass ratio was taken to be 1.5. Note from Table 3 that this eliminates values for the specific impulse of less than 1000 seconds for all the planets listed, and even for a specific impulse of 3000 seconds many missions cannot be completed. In Table 3, all maneuvers are assumed to be impulsive.

As in the case of the Hohmann trajectories, the minimum time trajectories can be modeled in three parts: 1) the departure from earth orbit, 2) the transit to the planet, and 3) the insertion into orbit about the planet. Figure 2 illustrates the geometry for the departure from earth orbit. There are two parameters that will determine the trip time to the planet. They are the velocity increment added to the circular orbit velocity, which is given by eq. (36) and the launch angle, $\phi_{\mathrm{i}}$. We have assumed that the impulse is applied tangent to the direction of motion. The launch angle is bounded by

$$
-\pi<\phi_{i}<\pi
$$


Table 3 Hohmann trajectory mass ratio Isp(sec)

\begin{tabular}{lrrrrrrrrr}
\hline \hline Planet & 500 & 1000 & 3000 & 10000 & 30000 & 100000 & 150000 & 200000 & 600000 \\
Mercury & 14.430 & 3.799 & 1.560 & 1.143 & 1.045 & 1.013 & 1.009 & 1.007 & 1.002 \\
Venus & 3.719 & 1.929 & 1.245 & 1.068 & 1.022 & 1.007 & 1.004 & 1.003 & 1.001 \\
Mars & 3.061 & 1.750 & 1.205 & 1.058 & 1.019 & 1.006 & 1.004 & 1.003 & 1.001 \\
Vesta & 5.815 & 2.411 & 1.341 & 1.092 & 1.030 & 1.010 & 1.006 & 1.004 & 1.001 \\
Ceres & 6.736 & 2.595 & 1.374 & 1.100 & 1.032 & 1.009 & 1.006 & 1.005 & 1.002 \\
Jupiter & 97.77 & 9.888 & 2.146 & 1.258 & 1.079 & 1.023 & 1.015 & 1.012 & 1.003 \\
Saturn & 33.50 & 5.788 & 1.795 & 1.192 & 1.060 & 1.018 & 1.012 & 1.009 & 1.003 \\
Uranus & 18.25 & 4.272 & 1.623 & 1.156 & 1.050 & 1.015 & 1.010 & 1.007 & 1.002 \\
Neptune & 21.03 & 4.586 & 1.611 & 1.164 & 1.052 & 1.015 & 1.010 & 1.008 & 1.003 \\
Pluto & 10.33 & 3.215 & 1.476 & 1.124 & 1.040 & 1.012 & 1.008 & 1.006 & 1.002 \\
\hline \hline
\end{tabular}

and the change in velocity, $\Delta v_{i}$, must be less than the total change in speed which is given by eq. (16), that is

$$
\Delta v=f I_{s p} g_{0} \ln M R
$$

where the factor $f$ must be less than one. The minimum transit time was found by incrementing through the range for the launch angle, and the velocity factor, to find the trajectories that reach the target planet. The trajectory with minimum travel time was found and tabulated. Appendix $B$ summarizes the equations used in the model.

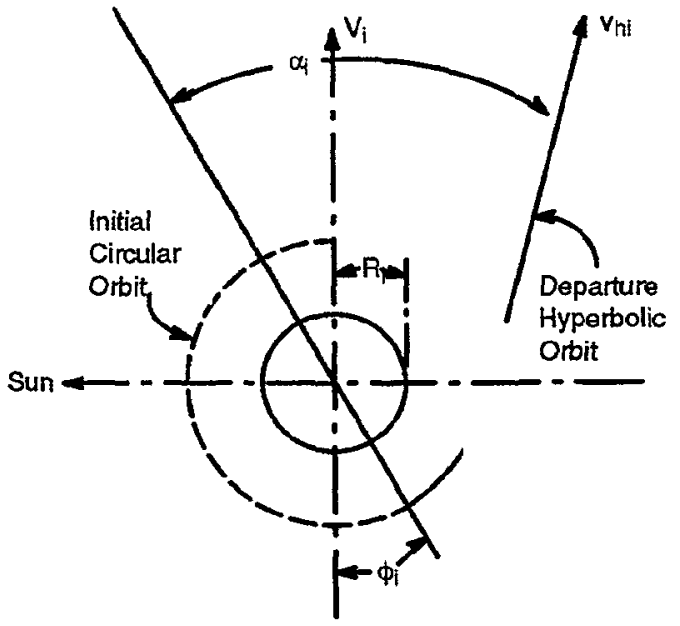

Fig. 2. Earth departure orbit parameters

Figures 3 and 4 illustrate the results of the analysis. For values of the specific impulse on the order of 500,000 seconds the trip times to Mars are on the order of one day. Trips to Mercury and Venus (Fig. 3) will still require a few days and trips to the asteroid belt will be several days. Hence, even for very high values of the specific impulse fusion rockets trip times to the inner planets will be longer than typical trip times around the earth now.
For trips to the outer planets (Fig. 4) trip times are never less than one week for high specific impulse fusion rockets, and to Pluto will be about one half year. Hence it will be helpful to make use of aeroassists and gravity assists whenever possible.

\section{Conclusions}

Inertial confinement fusion rockets can significantly increase the specific impulse over current values for chemical, and solid and gas core nuclear fission rockets. The performance increase will open up the solar system to settlement, and trade, but the trip times to the outer soLar system will be too long for quick trips to the outer solar system without making use of additional assists.

\section{References}

1. Forward, R. L., "Antiproton Annihilation Propulsion," Journal of Propulsion and Power, Vol. 1,No. 5, 1985, pp. 370-374.

2. Morgan, D. L., "Investigation of Matter-Antimatter Interactions for Possible Propulsion Applications," NASA CR-141356, Jan. 1975.

3. Vulpetti, G., "Antimatter Propulsion for Space Exploration," 36th Congress of the International Astronautical Federation, AIAA-85-491, New York, Oct. 1985.

4. Howe, S. D., and Metzger, J.D., "Survey of Antiproton-Based Propulsion Concepts and Potential Impact on a Manned Mars Mission," Los Alamos Preprint LA-UR-87-2191, Los Alamos National Lab., Los Alamos, NM, 1987.

5. Cassenti, B. N., "Conceptual Designs for Antiproton Space Propulsion Systems," Joumal of Propulsion and Power, Vol. 7, No. 3, 1991, pp. 368-373. 


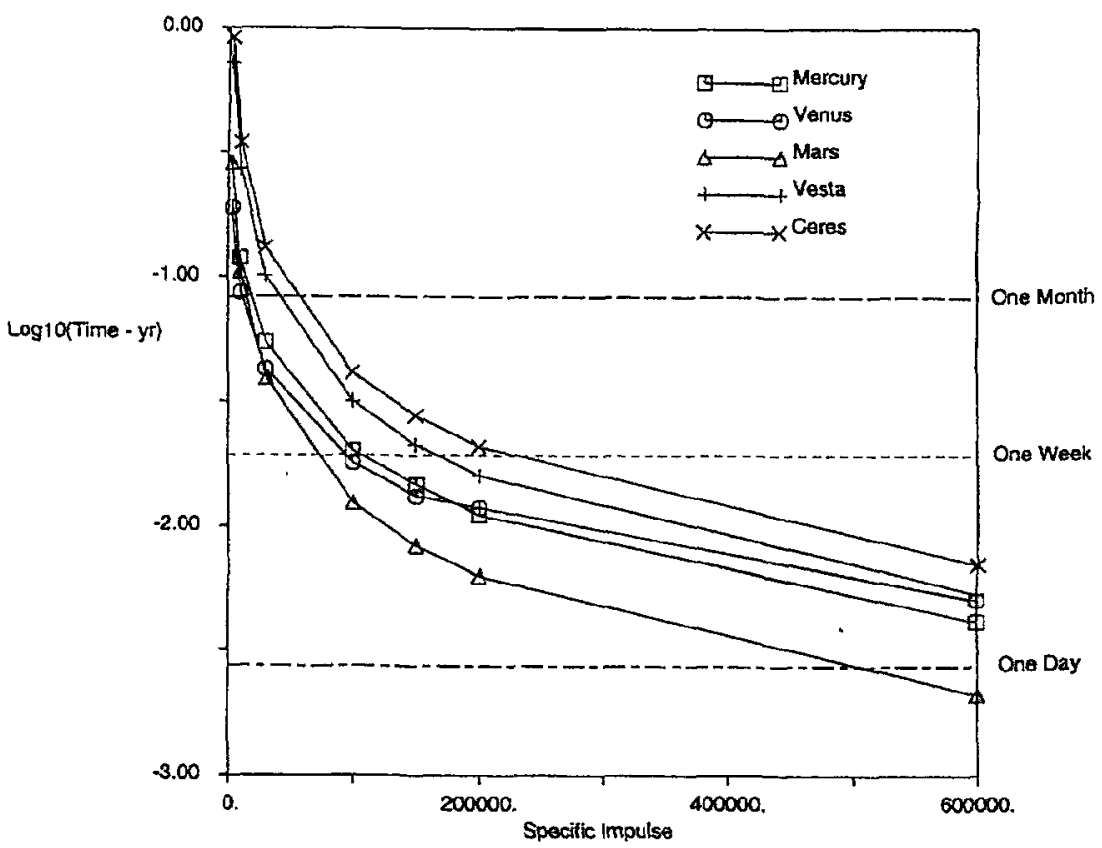

Fig. 3 Minimum transit time for a mass ratio of 1.5 to the inner planets

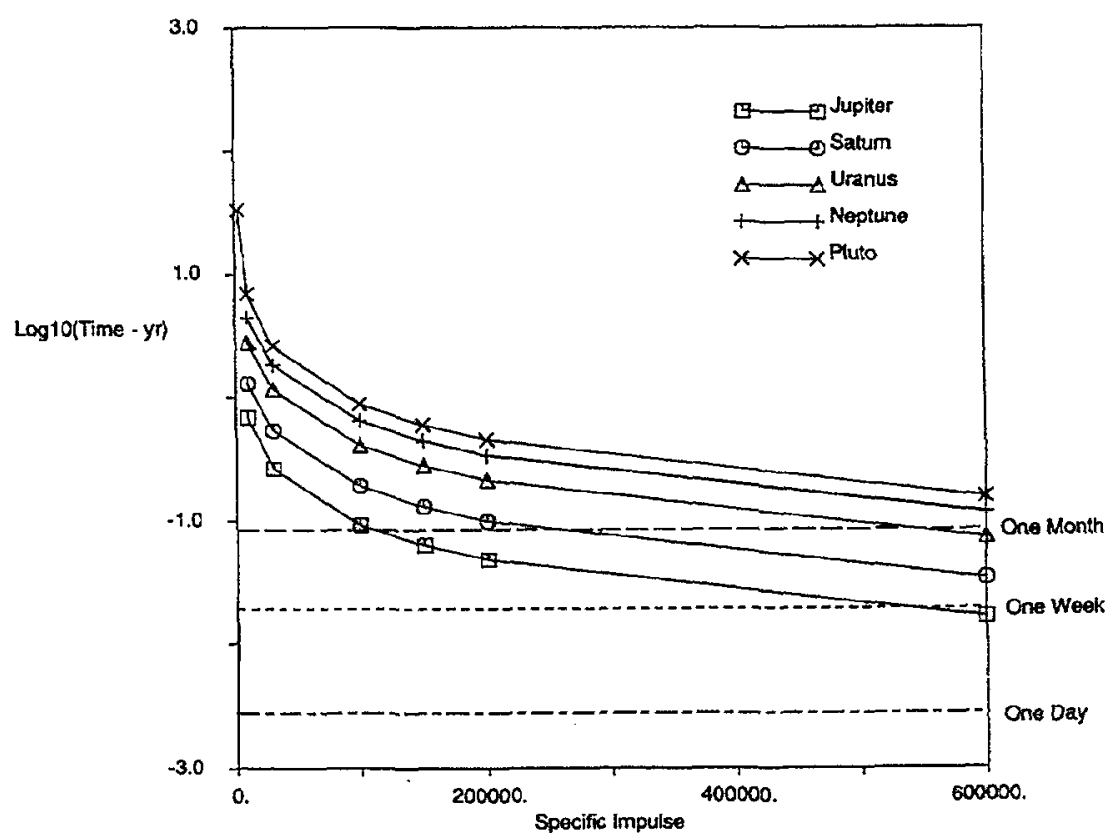

Fig. 4 Minimum transit time for a mass ratio of 1.5 to the outer planets

6. Cassenti, B. N., "Antimatter Propulsion for OTV Applications, Journal of Propulsion and Power, Vol. 1, No. 2, 1985, pp. 143-149.

7. Cassenti, B., Mannheim, P., and Gould, P., "Concepts for the Efficient Production and Storage of Antimatter," AIAA Paper 93-2031, 1993.
8. Cassenti, B. N., "Concepts for the Efficient Production of Antimatter," Eleventh Symposium Space Nuclear Power and Propulsion, AIP Conference Proceedings, Albuquerque, NM, Jan. 1994, pp. 1429-1434.

American Institute of Aeronautics and Astronautics 
9. Kammash, T. and Galbraith, D. L., "AntimatterDriven-Fusion Propulsion for Solar System Exploration," Journal of Propulsion and Power, Vol. 8, No. 3, 1992, pp. 644-649.

10. Lewis, R. A., Smith, G. A., Kanzlecter, R. J., and Newton, R., "Antiproton Based Microfusion," Fusion Technology, Vol. 20, Dec. 1991, pp. 1046-1050.

11. Lewis, R. A., Smith, G. A., Kanzlecter, R. J., and Newton, R., "An Antiproton Driver for Internal Confinement Fusion Propulsion," AIAA Paper 91-3618, Sept. 1991.

12. Lewis, R. A., Smith, G. A., Toothacker, W. S., and Kanzlecter, R. J., "An Antiproton Catalyst for Internal Confinement Fusion Propulsion," AIAA Paper 90-2760, July 1990.

13. Shmatov, M. L., "Ignition of Thermonuclear Microexplosions with Antimatter," A.F.IOFFE Physical Technical Institute, Preprint 1621, Academy of Sciences of Russia, St. Petersburg, 1993.

14. Takahashi, H., "Thoughts on the Muon Catalyzed Fusion Process for Antimatter Propulsion and for the Production of High Mass Number Nuclei," Antiproton Science and Technology, edited by B. W. Augenstein, Bonner, B. E., Mills, F. E., and Nieto, M. M., World Scientific, Singapore, 1988, pp. 603-619.

15. Morgan, D. L., "Annihilation of Antiprotons in Heavy Nuclei," Air Force Rocket Propulsion Lab., AFRPL TR 86-011, Edwards Air Force Base, Oct. 1986.

16. "Antiproton Induced Fission in $238 \mathrm{U}$ and $209 \mathrm{Bi}$," Physics at LEAR with Low Energy Antiprotons, Proceedings of the Fourth Low Energy Antiproton Ring Workshop, Villars-sur-Ollon, Switzerland, PS 177 Collaboration, 1988, pp. 793-796.

17. Cassenti, B. N., "High Specific Impulse Antimatter Rockets," AIAA Paper 91-2548, June 1991.

18. Hasegawa, A., "Magnetically Insulated Inertial Confinement Fusion: A New Approach to Controlled Thermonuclear Fusion," Physical Review Letters, Vol. 56, No. 2, 1986, pp. 139-142.

19. Karmmash, T., and Galbraith, D. L., "A High Gain Fusion Reactor Based on the Magnetically Insulated Inertial Confinement Fusion (MICF) Concept," Nuclear Fusion, Vol. 29, No. 7, 1989, pp. 1079-1099.
20. Kammash, T., and Galbraith, D. L., "Reaction Physics and Mission Capabilities of the MICF Reactor," Journal of Propulsion and Power, Vol. 6, No. 4, 1990, pp. 412-415.

21. Sakagani, Y., "Two Dimensional Distribution of Self-Generated Magnetic Fields Near the Laser Plasma Resonant Interaction Region," Physical Review Letters, Vol. 42, No. 13, 1979, pp. 839-842.

22. Max, C. E., Manheimer, W. M., and Thomson, J. J., "Enhanced Transport Across Laser Generated Magnetic Fields," Physics of Fluids, Vol. 21, No. 1, 1978, pp. 128-139.

23. Raven, A., Willi, O., and Rumsby, P. T., "Megagauss Magnetic Field Profiels in Laser Produced Plasmas," Physical Review Letters, Vol. 41, No. 8, 1978, pp. 554-557.

24. Subotowics, M., "Propulsion Concepts for Nuclear Matter Compression Energy and 'Cold' Fusion Energy Sources in Interstellar Flight," Acta Astronautica, Vol. 17, No. 8, 1988, pp. 937-942.

25. Cassenti, B. N., T. Rammash and D. L. Golbraith, "An Antiproton Catalyzed Inertial Fusion Propulsion System," Progress in Astronautics and Aeronautics, Vol. 167, edited by T. Kammash, pp. 75-88, 1995.

26. Nance, J. P., "Nuclear Pulse Propulsion," IEEE Transactions on Nuclear Science, Vol. NS-12, No. 1, 1965, pp. 177-182.

27. Martin, A. R., and A. Bond, "Nuclear Pulse Propulsion: A Historical Review of an Advanced Propulsion Concept," Journal of the British Interplanetary Society, Vol. 32, 1979, pp. 382-310.

28. Solem, J. D., "Medusa: Nuclear Explosive Propulsion for Interplanetary Travel," Journal of the British Interplanetary Society, Vol. 46, 1993, pp. 21-26.

29. Parlos, A. G., and J. D. Metzger, "Feasibility Study of a Contained Pulsed Nuclear Propulsion Engine," Journal of Propulsion and Power, Vol. 10, No. 2, 1994, pp. 269-278.

30. Cassenti, B. N., "A Contained Antiproton Catalyzed Pulse Nuclear Propulsion System," AIAA 95-2898, presented at the 31st AIAA/ASME/ SAE/ASEE Joint Propulsion Conference and Exhibit, July 10-12, 1995, San Diego.

31. Morgan, D. L., Jr., "Annihilation Localization in Gas-Core and Plasma Core Annihilation Rocket Engines," 39th Congress of the International Astronautical Federation,Paper IAA-88-554, Washington, DC, Oct. 1988. 
32. Rhodes, R., The Making of the Atomic Bomb, Simon and Schuster, New York, 1986, pp. 774-776.

33. Bate, R. R., D. D. Mueller and J. E. White: Fundamentals of Astrodynamics, Dover Publications, Inc., New York, 1971.

34. Beatty, J. K. and A. Charkin (editors): The New Solar System, 3rd edition, Cambridge University Press, New York, 1990.

\section{Appendix A - Solar System Parameters}

The characteristic solar system constants used in the evaluation of the orbits were:

$$
\begin{aligned}
\mathrm{Re}= & 6.378 \mathrm{E} 06 \mathrm{~m} \text { for the radius of the earth, } \\
\mathrm{au}= & 1.496 \mathrm{E} 11 \mathrm{~m} \text { for the astronomical unit, } \\
\mathrm{yr}= & 31.557 \mathrm{E} 06 \mathrm{~s} \text { for the length of the year, } \\
\mathrm{M} 0= & 1.989 \mathrm{E} 30 \mathrm{~kg} \text { for the mass of the sun, } \\
\mathrm{Me}= & 5.976 \mathrm{E} 24 \mathrm{~kg} \text { for the mass of the earth, } \\
\mathrm{g} 0= & 9.80665 \mathrm{~m} / \mathrm{s} \text { for the acceleration of gravity, and } \\
\mathrm{G}= & 6.67259 \mathrm{E}-11 \mathrm{~N} \mathrm{~m} / \mathrm{kg} \text { for the gravitational } \\
& \text { constant. }
\end{aligned}
$$

\begin{tabular}{|c|c|c|c|}
\hline Planet & $\frac{\text { Mass }}{\overline{M e}}$ & $\frac{\text { Radius }}{\overline{R e}}$ & $\frac{\text { Distance }}{\text { a.u. }}$ \\
\hline Mercury & 0.0553 & 0.382 & 0.3871 \\
\hline Venus & 0.8149 & 0.949 & 0.7233 \\
\hline Earth & 1.0000 & 1.000 & 1.0000 \\
\hline Mars & 0.1074 & 0.532 & 1.5327 \\
\hline Vesta & 0.000022 & 0.039 & 2.352 \\
\hline Ceres & 0.000134 & 0.0717 & 2.767 \\
\hline Jupiter & 317.938 & 11.209 & 5.2028 \\
\hline Saturn & 95.181 & 9.449 & 9.5388 \\
\hline Uranus & 14.531 & 4.007 & 19.1914 \\
\hline Neptune & 17.135 & 3.883 & 30.0511 \\
\hline Pluto & 0.0022 & 0.180 & 39.5294 \\
\hline
\end{tabular}

The data used for the planets were: ${ }^{34}$

Appendix B - Solar Transit Orbit Model

The total available speed change is given by

$$
\Delta v=I_{s p g_{0}} \ln M R
$$

The initial speed change from the circular orbit about earth to the hyperbolic escape orbit is a fraction, $\mathrm{f}$, of the total available speed change or

$$
\Delta v_{i}=f \Delta v
$$

where $0 \leq f \leq 1$.

The fraction, $f$, and the launch angle, $\phi_{1}$ in Fig. 1, (which is bounded by $-\pi \leq \phi_{i} \leq \pi$ ) are incremented over the available range.

The departure orbit can now be found.

$$
v_{p i}=\Delta v_{i}+\sqrt{\frac{G M_{i}}{R_{i}}} \text { is the perigee velocity. (B-3) }
$$$$
E_{i}=\frac{1}{2} v_{p i}^{2}-\frac{G M_{i}}{R_{i}} \text { is the specific energy. }
$$

$v_{h i}=\sqrt{2 E_{i}}$ is the hyperbolic excess velocity.

$h_{i}=v_{p i} R_{i}$ is the specific mom.

$$
P_{i}=\frac{h_{i}^{2}}{G M_{i}} \text { is the semi-parameter. }
$$

$$
e_{i}=\sqrt{1-\frac{P_{i}}{a_{i}}} \text { is the eccentricity. }
$$

$$
\alpha_{i}=\cos ^{-1}\left(\frac{1}{e_{i}}\right) \text { is the departure angle. }
$$

The solar transfer orbit can now be evaluated (see Fig. B-1).

$$
v_{i}=\sqrt{v_{h i}^{2}+V_{i}^{2}+2 V_{i} v_{h i} \cos \left(\alpha_{i}-\phi_{i}\right)}
$$

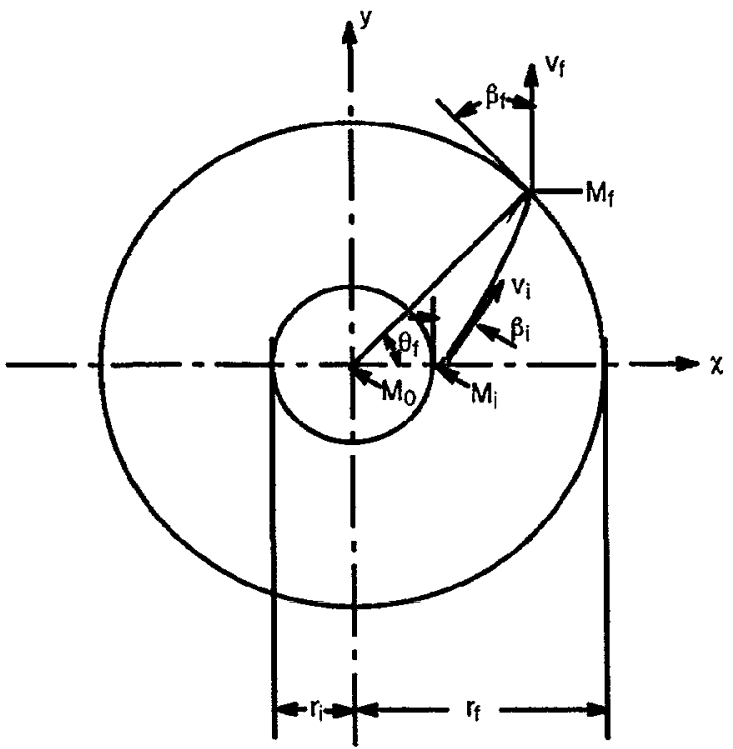

Fig. B-1. Solar transit orbit parameters 
is the initial solar orbit velocity, where

$$
\begin{aligned}
& V_{i}=\frac{\sqrt{G M_{i}}}{r_{i}} \text { is the earth orbit velocity. } \\
& \beta_{i}=\operatorname{Tan}^{-1}\left[\frac{v_{h i} \sin \left(\alpha_{i}-\phi_{i}\right)}{V_{i}+\nu_{h i} \cos \left(\alpha_{i}-\phi_{i}\right)}\right]
\end{aligned}
$$

is the departure angle.

$$
\begin{aligned}
& E_{0}=\frac{1}{2} v_{i}^{2}-\frac{G M_{0}}{r_{i}} \text { is the specific energy } \\
& a_{0}=-\frac{G M_{0}}{2 E_{0}} \text { is the semi-major axis } \\
& h_{0}=r_{i} v_{i} \cos \beta_{i} \text { is the specific }
\end{aligned}
$$

angular momentum

$$
\begin{aligned}
& e_{0}=\sqrt{1-\frac{p_{0}}{a_{0}}} \text { is the eccentricity } \\
& v_{f}=\sqrt{2\left(E_{0}+\frac{G M_{0}}{r_{f}}\right)}
\end{aligned}
$$

is the final solar velocity

$$
\theta_{0}=\cos ^{-1}\left[\frac{1}{e_{0}}\left(\frac{p_{0}}{r_{i}}-1\right)\right]
$$

is the perihelion location

$$
\theta_{f}=\theta_{0}+\cos ^{-1}\left[\frac{1}{e_{0}}\left(\frac{p_{0}}{r_{f}}-1\right)\right]
$$

is the final angular location

$$
B_{f}=\cos ^{-1}\left(\frac{h_{0}}{r_{f} \nu_{f}}\right)
$$

These models are summarized below.

The arrival orbit parameters can now be found.

$$
v_{h f}=\sqrt{v_{f}^{2}+V_{f}^{2}-2 v_{f} V_{f} \cos \beta_{f}}
$$

where

$$
v_{f}=\sqrt{\frac{G M_{f}}{R_{f}}}
$$

is the hyperbolic excess velocity

$$
E_{f}=\frac{1}{2} v_{h f}^{2} \text { is the specific energy }
$$

$v_{p f}=\sqrt{2\left(E_{f}+\frac{G M_{f}}{R_{f}}\right)}$ is the periapsis velocity (B-24)

$$
\Delta v_{f}=v_{p f}-\sqrt{\frac{G M_{f}}{R_{f}}} \text { is the change in }
$$

speed to go onto the circular parking orbit the total $\Delta v_{i}+\Delta v_{f}$ can now be compared to $\Delta v$ in eq.(B-1). Values of $f$ and $\beta_{i}$ can now be found by interpolation.

The transit time can now be evaluated by using any standard method of celestial mechanics. 